It is interesting to compare (14) with the solution obtained by Crout. In footnote 2, five significant figures were kept in the computation and the solution of (11) was given as

$$
\text { (0.15942, 0.14687, 0.11261, 0.060806.). }
$$

The equivalent matrices would be in better form of course, if a more careful calculation of (12) were made. Thus, keeping five significant figures in the solution of (6), a pseudo diagonalizing operator was obtained for which the equivalent system was solved to nine decimal accuracy in four iterations.

To illustrate the methods of Section IV, an approximate value of $Q_{1}$ in (9) was chosen to accelerate the solution of (13). To maintain a reasonable number of significant figures, the coefficients of (13) were mentally rounded off to two decimal points. By inspection, therefore, the improving operator, $Q_{1}$, becomes

$$
Q_{1}=\left(\begin{array}{rrrr}
.89 & -.04 & .26 & .23 \\
.13 & 1.01 & -.38 & .06 \\
-.10 & -.18 & .96 & -.09 \\
.11 & .21 & .02 & 1.02
\end{array}\right)
$$

The equivalent system formed by multiplying (13) by (16) required only five iterations to yield (14).

Acknowledgment: The author is indebted to Lyman B. Stewart, Watertown Arsenal, for examination of the computational feasibility of the procedures.

\title{
PLASTIC DESIGN OF BEAMS AND PLANE FRAMES FOR MINIMUM MATERIAL CONSUMPTION*
}

BY JACQUES HEYMAN (Brown University)**

\begin{abstract}
This paper is concerned with the design of plane frames in such a way that the material consumption is a minimum. The method of solution is to set up linear inequalities for the variables involved, and to solve these inequalities by the Dines ${ }^{1}$ method. Three slightly different classes of problem are treated; collapse design under fixed loads, collapse design under varying loads, and shakedown design under varying loads. Illustrative examples of each are given.

1. Introduction. The problem discussed in this paper may be stated as follows: Given the geometrical configuration of a frame which is acted upon by specified loads, how should the various members of the frame be proportioned so that the total material consumption is as small as possible? The type of frame to be considered consists of straight members, all in the same plane. Between joints, the assumption is made that any one member has a uniform cross-section. The moment-curvature curve for any cross-section will be taken to have the general form shown in Fig. 1, where a limit

*Received March 7, 1950. This paper is a shortened version of Technical Report A11-45 prepared for the Office of Naval Research under contract N7onr-358.

**Now Fellow of Peterhouse College, Cambridge, England.

1. Dines, L. L. Systems of linear inequalities. Ann. Math. (2) 20, 191 (1918-1919).
\end{abstract}


moment $M$ is attained at a finite curvature such that any further bending is accompanied by no increase in $M$. This limit value $M$ is known as the full plastic moment, and the section at which it occurs is called a plastic hinge. It will be assumed that the joints connecting the members of the frame are sufficiently rigid for the full plastic moments to be developed (if necessary) at those joints.

The total material consumption $x$ for a given frame is given by the proportionality

$$
x \propto \sum_{i=1}^{N} M_{i} l_{i},
$$

where $M_{i}$ is the full plastic moment of the $i^{t h}$ member of the frame, and $l_{i}$ is its length. This linearization of the material consumption is not strictly correct, but for the purpose of developing suitable methods for design, it will be assumed that a continuous linear range of sections is available.

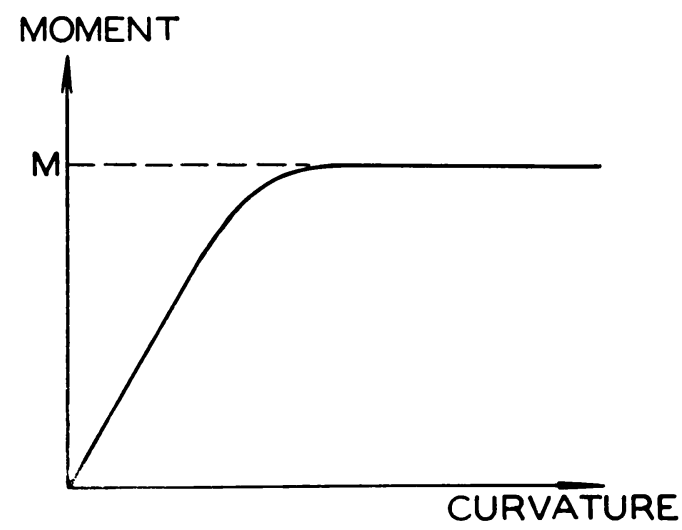

FIG. 1

The general state of a frame of $n$ redundancies can be expressed as the sum of one arbitrary fixed equilibrium state and $n$ linearly independent residual states. By a state is meant some distribution of bending moment, so that a state in equilibrium with the applied loads is any bending moment distribution such that equilibrium is attained. A residual state satisfies equilibrium when the applied loads on the frame are zeroThus, confining attention to any one cross-section in a frame, the bending moment there may be expressed as

$$
M^{*}+c_{1} M_{1}^{\prime}+c_{2} M_{2}^{\prime}+\cdots+c_{n} M_{n}^{\prime},
$$

where $M^{*}$ is the equilibrium bending moment at the section, and $M_{1}^{\prime} \cdots M_{n}^{\prime}$ are the linearly independent residual moments. Suppose the full plastic moment at the section (as yet undetermined) is $M$. Then since the total bending moment cannot exceed the value $M$,

$$
-M \leq M^{*}+c_{1} M_{1}^{\prime}+c_{2} M_{2}^{\prime}+\cdots+c_{n} M_{n}^{\prime} \leq M
$$

and continued inequalities similar to (3) may be written for every critical section of the frame. Only concentrated loads are considered in this paper, so that shear forces are discontinuous at the joints and under the loads, and are constant elsewhere. This 
means that the bending moments in the frame are linear, and critical sections can occur only at the joints and under the loads. Writing down the inequalities (3) for these sections, the problem may be solved by Dines' method, and the ideas are best presented by means of examples. On account of the limitations of space, these examples will deal with the design of beams; the design of plane frames introduces no new ideas but in general entails rather more arithmetical labour. (In the full report, of which the present paper is a shortened version, a rectangular portal frame is treated).

2. Example 1 (fixed loads). Consider the two-span continuous beam on three supports carrying the central fixed loads $P_{1}$ and $P_{2}\left(P_{1} \geq P_{2}\right)$ as shown in Fig. 2. Since the sys-

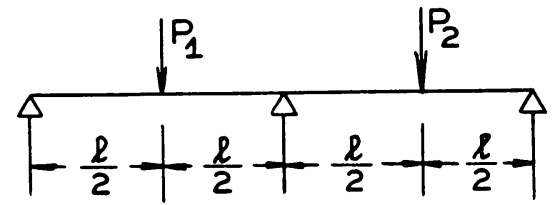

Fig. 2

tem has one redundancy, the plastic behaviour can be represented as the sum of an arbitrary fixed equilibrium state and one residual state. These may be taken as the two bending moment distributions in Fig. 3, where the factor $c$ corresponds to the

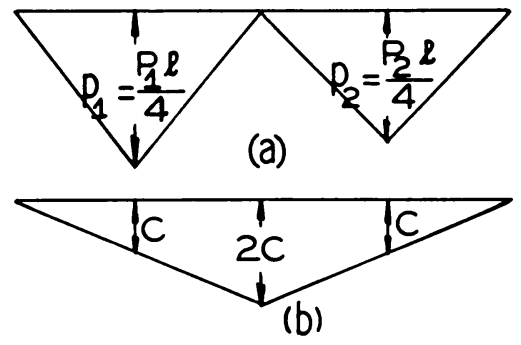

FIG. 3

arbitrary multiplying factors on the residual moments in expression (2). Denoting the full plastic moments of the two spans by $M_{1}$ and $M_{2}$ (the values being as yet undetermined), the condition that the total bending moments at the critical points should be less than or equal to the full plastic moments at those points gives the following inequalities:
(1) under the load $P_{1}$,$$
-M_{1} \leq p_{1}+c \leq M_{1}
$$
(2) at the central support,$$
-M_{1} \leq 2 c \leq M_{1}
$$$$
-M_{2} \leq 2 c \leq M_{2}
$$
(3) under the load $P_{2}$,
$-M_{2} \leq p_{2}+c \leq M_{2}$,

where $p_{1}$ for example is given by $p_{1}=P_{1} l / 4$, the "free" bending moment shown in Fig. $3(\mathrm{a})$. The two inequalities for the central support are obtained by considering respec- 
tively points slightly to the left and to the right of the support, since it is not known $a$ priori whether $M_{1} \gtrless M_{2}$.

For the purposes of calculation, each continued inequality in (4) may be expressed as two simple inequalities, and the set (4) rewritten:

$$
\begin{array}{rlrl}
c+p_{1}+M_{1} & \geq 0, & -c-p_{1}+M_{1} \geq 0, \\
+\frac{M_{1}}{2} \geq 0, & -c & +\frac{M_{1}}{2} \geq 0, \\
c \quad+\frac{M_{2}}{2} \geq 0, & -c \quad+\frac{M_{2}}{2} \geq 0, \\
c+p_{2}+M_{2} \geq 0, & -c-p_{2}+M_{2} \geq 0 .
\end{array}
$$

For the present purposes, the actual value of $c$ in the set (5) is of no interest, so long as it is known that a value of $c$ exists such that each inequality can be satisfied. Dines has shown that necessary and sufficient conditions for the existence of such a value of $c$ are obtained if every inequality in the set (5) with a coefficient of +1 for $c$ is added to every inequality with a coefficient of -1 for $c$. If these additions are performed, $c$ will be eliminated, and the resulting set of inequalities ( $4 \times 4=16$ in this example) provide the necessary and sufficient conditions for the existence of $c$.

In the actual operation, it is found that some inequalities are redundant; for example, adding the first and second of set ( $\tilde{5})$ gives $M_{1} \geq 0$, while the second and third give $M_{1} \geq \frac{2}{3} p_{1}$, so that $M_{1} \geq 0$ is redundant. The five remaining inequalities out of the possible sixteen are

$$
\begin{aligned}
-p_{1}+\frac{3}{2} M_{1} & \geq 0, \\
-p_{1}+M_{1}+\frac{1}{2} M_{2} & \geq 0, \\
-p_{1}+p_{2}+M_{1}+M_{2} & \geq 0, \\
-p_{2}+\frac{1}{2} M_{1}+M_{2} & \geq 0, \\
-p_{2}+\frac{3}{2} M_{2} & \geq 0 .
\end{aligned}
$$

The material consumption parameter $x$ will now be introduced into set (6). Since the two beams have equal spans, $x$ may be taken as (see (1))

$$
x=M_{1}+M_{2} .
$$

$M_{1}$ may be replaced in set (6) by $\left(x-M_{2}\right)$, giving, upon slight rearrangement,

$$
\begin{aligned}
-M_{2}+x-\frac{2}{3} p_{1} & \geq 0, \\
-M_{2}+2 x-2 p_{1} & \geq 0, \\
M_{2}+x-2 p_{2} & \geq 0, \\
M_{2}-\frac{2}{3} p_{2} & \geq 0,
\end{aligned}
$$


together with

$$
x \geq\left(p_{1}-p_{2}\right) .
$$

Now for the problem of determining the minimum value of $x$, the value of $M_{2}$ is not required, and Dines' method may be employed again on set (8) to eliminate $M_{2}$, giving

$$
\begin{aligned}
& x \geq \frac{1}{3} p_{1}+p_{2}, \\
& x \geq \frac{1}{3} p_{1}+\frac{1}{3} p_{2}, \\
& x \geq \frac{2}{3} p_{1}+\frac{2}{3} p_{2}, \\
& x \geq p_{1}+\frac{1}{3} p_{2} .
\end{aligned}
$$

Inequality (9) and the second of (10) are obviously redundant, and for $p_{1} \geq p_{2}$ (as assumed), set (10) reduces to

$$
x \geq p_{1}+\frac{1}{3} p_{2},
$$

the other two inequalities also being redundant. This single inequality (11) is a necessary and completely sufficient condition that values of $M_{1}, M_{2}$, and $c$ can be found to satisfy the original set (4). Since it is required that $x$ should be as small as possible, the equality sign will be taken in (11), so that

$$
x=p_{1}+\frac{1}{3} p_{2}
$$

that is, the existence condition is just satisfied. It is to be expected that unique values of $M_{1}, M_{2}$, and $c$ will be derived, and this is in fact the case. Inequality (11) was obtained by the addition of the second and fourth of set (8); the second of (8) states that $M_{2} \leq \frac{2}{3} p_{2}$, for the minimum value of $x$ given by Eq. (12), while the fourth gives $M_{2} \geq$ $\frac{2}{3} p_{2}$. Hence

$$
M_{2}=\frac{2}{3} p_{2}, \quad M_{1}=p_{1}-\frac{1}{3} p_{2}
$$

and, by going back to the original set (5),

$$
2 c=-\frac{2}{3} p_{2}=-M_{2} .
$$

The bending moment distribution resulting from the analysis is shown in Fig. 4, which

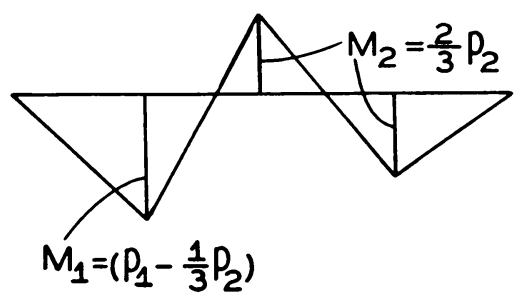

FrG. 4

shows that plastic hinges are formed at all three of the critical points. Physically, this number of hinges is sufficient for the structure to become a mechanism and hence to collapse. 
3. Example 2 (variable loads, collapse design). Consider the same beam shown in Fig. 2 but with the loads varying between the limits

$$
-Q_{1} \leq P_{1} \leq Q_{1}, \quad-Q_{2} \leq P_{2} \leq Q_{2}, \quad Q_{1} \geq Q_{2} .
$$

The same set of inequalities (5) may be written down containing the parameters $p_{1}$ and $p_{2}$. The sixteen inequalities resulting from the elimination of $c$ must be examined in the light of the fact that $p_{1}$ and $p_{2}$ vary, and the worst values of $p_{1}$ and $p_{2}$ (i.e., $\pm q_{1}$, $\pm q_{2}$ ) inserted in each inequality. After removal of redundant inequalities, the set becomes

$$
\begin{aligned}
-q_{1}+\frac{3}{2} M_{1} & \geq 0, \\
-q_{1}+M_{1}+\frac{1}{2} M_{2} & \geq 0, \\
-q_{1}-q_{2}+M_{1}+M_{2} & \geq 0, \\
-q_{2}+\frac{1}{2} M_{1}+M_{2} & \geq 0, \\
-q_{2}+\frac{3}{2} M_{2} & \geq 0 .
\end{aligned}
$$

Operating on this set as before to find the minimum value of $x$, it is found that the third of (16) is the controlling inequality, giving

$$
x=q_{1}+q_{2} .
$$

This is one of the few examples where unique values of $M_{1}$ and $M_{2}$ are not generated by working back through the successive sets of inequalities; instead, ranges are found as follows:

$$
\begin{gathered}
M_{1}+M_{2}=x=q_{1}+q_{2}, \\
\left(q_{1}+\frac{1}{3} q_{2}\right) \geq M_{1} \geq \frac{2}{3} q_{1}, \\
2 q_{2} \geq M_{2} \geq \frac{2}{3} q_{2}, \\
\left(\frac{1}{3} q_{1}+q_{2}\right) \geq M_{2} .
\end{gathered}
$$

As a specific example, suppose $q_{1}=q_{2}=q$. Then

$$
M_{1}+M_{2}=2 q, \quad \frac{4}{3} q \geq M_{1} \geq \frac{2}{3} q, \quad \frac{4}{3} q \geq M_{2} \geq \frac{2}{3} q,
$$

and any values of $M_{1}$ and $M_{2}$ satisfying (19) will give a constant material consumption. [It is perhaps of interest to note that if $x=\Sigma\left(M^{n} l\right)$, where $n<1$, the minimum material consumption is given by $M_{1}=2 M_{2}=\frac{4}{3} q$ (or vice versa), the worst case occurring for $M_{1}=M_{2}=q$. An asymmetrical solution is obtained for what appears to be a completely symmetrical problem. In practice, for $n=0.6$, which is a reasonable value for the exponent, the worst error due to the linearized material consumption amounts to less than $2 \%$ in this example].

4. Example 3 (variable loads, shakedown design). If, after a certain arbitrary time, a structure subjected to varying loads resists all further changes of load in a purely 
elastic manner, then the structure is said to shake down under the given loading system. It may be shown ${ }^{2}$ that a necessary and sufficient condition for shakedown to occur is that a sum of residual states can be found such that when it is added to the elastic response of the structure, the moment at any cross-section is less in magnitude than the full plastic moment at that section, that is (cf. Eq. (3)),

$$
-M \leq M_{\bullet}+c_{1} M_{1}^{\prime}+c_{2} M_{2}^{\prime}+\cdots c_{n} M_{n}^{\prime} \leq M,
$$

where $M_{e}$ is the elastic solution for the problem under the given loads. Now, as the loads on the frame vary, $M_{e}$ will vary between limits, say $M_{e}^{\max }$ and $M_{e}^{\min }$, so that (20) may be replaced by the two inequalities

$$
\begin{aligned}
& M_{a}^{\max }+c_{1} M_{1}^{\prime}+c_{2} M_{2}^{\prime}+\cdots+c_{n} M_{n}^{\prime} \leq M, \\
& \quad-M \leq M_{e}^{\min }+c_{1} M_{1}^{\prime}+c_{2} M_{2}^{\prime}+\cdots+c_{n} M_{n}^{\prime} .
\end{aligned}
$$

The elastic solution of a given structure presents no formal problem, although for highly redundant frames the numerical labour may be heavy. The bending moment at any cross-section may be expressed in terms of the given loads and the flexural rigidity (B) of the various portions of the frame, so that the elastic parameters (flexural rigidities) used in the solution are not the same as the plastic parameters (full plastic moments). However, once a certain class of cross-section has been decided upon, an empirical relationship may be plotted connecting the two, so that if a full plastic moment is given, it is simple to read off the corresponding flexural rigidity of the section, and vice versa.

In order to work the following example analytically (and it should be stressed that any given relationship will not lead to more complicated results), it will be assumed that

$$
B=k M^{4 / 3},
$$

where $k$ is a numerical constant. The factor of $4 / 3$ expresses the fact that approximately geometrically similar sections are involved.

Consider the same beam as in the previous examples (see Fig. 2) with the loads $P_{1}$ and $P_{2}$ varying arbitrarily between the limits

$$
0 \leq P_{1} \leq P, \quad 0 \leq P_{2} \leq 2 P .
$$

If the flexural rigidities of the two spans are $B_{1}$ and $B_{2}$, the elastic bending moments at the critical points are:

(1) under $P_{1}$,

$$
M_{\bullet 1}=\frac{1}{8}\left[\frac{p_{1}\left(5 B_{2}+8 B_{1}\right)-p_{2}\left(3 B_{1}\right)}{\left(B_{1}+B_{2}\right)}\right],
$$

(2) at the central support,

$$
M_{\bullet 2}=-\frac{1}{8}\left[\frac{p_{1}\left(3 B_{2}\right)+p_{2}\left(3 B_{1}\right)}{\left(B_{1}+B_{2}\right)}\right],
$$

(3) under $P_{2}$,

$$
M_{e 3}=\frac{1}{8}\left[-\frac{p_{1}\left(3 B_{2}\right)+p_{2}\left(5 B_{1}+8 B_{2}\right)}{\left(B_{1}+B_{2}\right)}\right] \text {. }
$$

${ }^{2}$ Neal, B. G., The behaviour of continuous beams and plane frames under repeated loading, Technical Report A11-32 to the Office of Naval Research under contract N7onr-358. 
Hence as $P_{1}$ and $P_{2}$ vary between their limits,

$$
\begin{array}{ll}
M_{e 1}^{\max }=\frac{p}{8}\left[\frac{5 B_{2}+8 B_{1}}{B_{1}+B_{2}}\right], & M_{e 1}^{\min }=-\frac{p}{8}\left[\frac{6 B_{1}}{B_{1}+B_{2}}\right], \\
M_{e 2}^{\max }=0, & M_{e 2}^{\min }=-\frac{p}{8}\left[\frac{3 B_{2}+6 B_{1}}{B_{1}+B_{2}}\right], \\
M_{e 3}^{\max }=\frac{p}{8}\left[\frac{10 B_{1}+16 B_{2}}{B_{1}+B_{2}}\right], & M_{e 3}^{\min }=-\frac{p}{8}\left[\frac{3 B_{2}}{B_{1}+B_{2}}\right] .
\end{array}
$$

These values of moments are non-linear in the values of $B$ even for this simple example, and in any case the relationship between $B$ and $M$ (the full plastic moment) is not expressible analytically in general, so that an iterative numerical method will be developed. The general method of solution may be tabulated as follows.

1. Assign flexural rigidities to the various members of the frame, specifying these rigidities in terms of one unknown.

2. The elastic solution may be obtained, and the limits of the bending moments at critical sections calculated as the loads on the frame vary.

3. Forgetting that flexural rigidities have already been specified, use these elastic limit moments as a state of the frame to which must be added some sum of residual states such that the bending moment at any section is less in magnitude than the full plastic moment.

4. Set up the usual inequalities, and solve the problem by Dines' method, which will give the actual full plastic moments necessary for minimum material consumption.

5. From these full plastic moments, calculate (either by means of an empirical curve or by some relationship such as Eq. (22)) the flexural rigidities of the various members. If these rigidities are far removed from those originally assumed, obtain an elastic solution with the new values, and rework the problem.

Continuing with the two-span beam example, if it is assumed that

$$
B_{2}=2 B_{1}
$$

(since the right hand span has to support twice as much load as the left hand), then, working to the rules given above, it is found that

$$
x=\frac{7}{3} p=2.33 p, \quad M_{1}=\frac{2}{3} p=0.67 p, \quad M_{2}=\frac{5}{3} p=1.67 p .
$$

Hence for the next trial, from Eq. (22), take

$$
\frac{B_{2}}{B_{1}}=\left(\frac{M_{2}}{M_{1}}\right)^{4 / 3}=(2.5)^{4 / 3}=3.4 .
$$

This ratio of flexural rigidities gives

$$
x=2.68 p, \quad M_{1}=0.78 p, \quad M_{2}=1.90 p,
$$

and the next trial, although $B_{2} / B_{1}$, is reduced to 3.25 , gives these same values of $M_{1}$ and $M_{2}$. 
5. Conclusion. The methods presented above have been applied to more complicated frames and readily give the required solutions for collapse design under constant or varying loads. For shakedown design, the iterative numerical method converges fairly rapidly. It may be found easier for highly redundant frames to obtain a new elastic solution at each stage using the numerical values obtained from the previous analysis, since an analysis with numerically unspecified flexural rigidities is extremely tedious. For all three types of design, the introduction at an early stage of the numerical values of the loads simplifies the work greatly, since it will be found that a large proportion of the inequalities generated become redundant and can be ignored.

Only examples of concentrated loads on straight members of uniform cross-section between joints have been examined, making it possible to pick by inspection the critical cross-sections. However, the basic ideas are not altered by the introduction of other variables; the analysis will be more complicated, but aids to calculation may be introduced which leave the basic problem unchanged.

\title{
THE METHOD OF CHARACTERISTICS APPLIED TO PROBLEMS OF STEADY MOTION IN PLANE PLASTIC STRESS*
}

\author{
By P. G. HODGE, JR. (University of California at Los Angeles)
}

A method is outlined for obtaining the stress, strain, and thickness distribution in a thin sheet which is strained plastically in its plane. For the particular case of steady motion, a method is given for obtaining directly the final solution to certain types of boundary value problems. A step by step procedure is indicated for the general case of non-steady motion.

1. Introduction. This paper is concerned with the stress and strain distribution in a thin sheet which is strained plastically in its plane, under conditions of plane stress. It will be shown that three types of problems may be distinguished. In certain special cases the stress distribution may be found independently of the velocity or thickness by solving three equations in as many unknowns. For general steady motion problems it will be necessary to solve six equations simultaneously for three stress components, two velocity components, and the thickness. These equations will be stated in Sec. 2, and reduced to a system of five first order, quasi-linear differential equations under the assumption of initial isotropy. For suitable boundary conditions, it will be possible to find the final stress, strain, and thickness distribution of the material directly, using the method of characteristics. The details of this method of solution will be described in Secs. 3 and 4. Finally, in Sec. 5, a step-by-step procedure for solving problems of non-steady motion will be briefly indicated.

2. Basic equations. Let the sheet be referred to a set of Cartesian axes such that the $x, y$ plane coincides with the middle surface, and let $z= \pm \frac{1}{2} h(x, y)$ be the equations of the bounding surfaces. Under the assumptions of generalized plane stress, the only non-vanishing averaged stress components are $\sigma_{x}, \sigma_{y}$, and $\tau_{x y}$. These components must satisfy the equations of equilibrium

\footnotetext{
*Received March 29, 1950.
} 